\title{
Role of the extracellular matrix in skin aging and dedicated treatment - State of the art
}

\author{
Adele Sparavigna \\ Derming, Clinical Research and Bioengineering Institute, Milan 20159, Italy. \\ Correspondence to: Dr. Adele Sparavigna, Derming, Clinical Research and Bioengineering Institute, Milan 20159, Italy. \\ E-mail: adele.sparavigna@yahoo.it
}

How to cite this article: Sparavigna A. Role of the extracellular matrix in skin aging and dedicated treatment - State of the art. Plast Aesthet Res 2020;7:14. http://dx.doi.org/10.20517/2347-9264.2019.73

Received: 13 Dec 2019 First Decision: 25 Feb 2020 Revised: 3 Mar 2020 Accepted: 12 Mar 2020 Published: 20 Mar 2020

Science Editors: John Yousif, Kai O. Kaye Copy Editor: Jing-Wen Zhang Production Editor: Tian Zhang

\begin{abstract}
The extracellular matrix (ECM) occupies the space between cell and cell, and serves as a sort of intranet which connects the whole organism. Current research is focused on the ECM and, it is now possible to develop increasingly effective strategies for the prevention and treatment of degenerative diseases and even, cutaneous ageing. In fact, the most advanced anti-aging treatments are those that regenerate the ECM, which is now regarded as the main player in the physical support of, and exchange with and between cells of nutrients, cellular mediators and growth factors.
\end{abstract}

Keywords: Collagen, elastin, hyaluronic acid, extracellular matrix, matrisome

\section{INTRODUCTION}

Skin aging is a complex and unavoidable biological phenomenon that starts in the third decade of life ${ }^{[1]}$. The aging processes is determined by a combination of intrinsic (chronological, hormonal and genetic) and extrinsic factors. The latter can be further divided into behavioural factors like sun exposure (responsible for photo-aging), cigarette smoking, dietary habits, alcohol intake, drug abuse and environmental factors such as pollution, weather, and humidity. Between individuals, genetics, as well as lifestyle habits, are all different. Therefore, aging at large, and skin aging in particular, are extremely variable ${ }^{[2]}$. Skin aging is a dynamic process that results in structural alterations of soft and bony tissues ${ }^{[3]}$. The silhouette of the face and body change as a result of atrophy of its various constituent layers: from skin to subcutaneous adipose tissue including loss of muscle mass and, to a lesser extent, resorption of bone, wrinkles, hypotonicity, lipoatrophy, sarcopenia and volume displacement may result. Furthermore, to counteract atrophy, a

Cc) (7) The Author(s) 2020. Open Access This article is licensed under a Creative Commons Attribution 4.0 International License (https://creativecommons.org/licenses/by/4.0/), which permits unrestricted use, sharing, adaptation, distribution and reproduction in any medium or format, for any purpose, even commercially, as long as you give appropriate credit to the original author(s) and the source, provide a link to the Creative Commons license, and indicate if changes were made. 
permanent muscle tone is established to neutralize incorrect relaxation of the skin through compensating mechanisms ${ }^{[4]}$.

\section{EXTRACELLULAR MATRIX AGING MECHANISMS}

Over time, various body structures lose function in an unpredictable sequence. The aging process is thus not gradual nor uniform, and aging of the facies becomes even more complex due to the presence of mimetic muscles connected to the skin. Prevention and treatment strategies must therefore account for the above. The extracellular matrix (ECM) provides a commonality amongst these intricate processes and has emerged as an area of research focuse for the development of more effective strategies against the cutaneous signs of aging ${ }^{[5]}$.

There are several molecular mechanisms underlying the aging process. Although still not completely undisclosed, such knowledge is very important clinically and is the focus of many current studies. The main extrinsic factor responsible is still photodamage, which causes the release of collagenases and neutrophil elastases that result in fragmentation of structural and tonic proteins. Additionally, the cellular responses of autophagy and Ubiquitin Proteasome systems are slowed. Current strategies of ECM remodeling are thus based on eliminating cellular debris and the stimulation of neocollagenesis, elastogenesis and glycosaminoglycans production through the use of various peptides and other active agent ${ }^{[6-8]}$. As far as we know, the main mechanism involved in preventing ECM changes is to keep free radical (i.e., ROS, reactive oxygen species) production under control. ROS are characterized by the presence of an unpaired electron, which is responsible for their instability and reactivity towards chemical structures to which they bind to capture another electron. In turn, this process generates unstable molecules and promotes chain reactions that ultimately, lead to functional and structural damage both within cells and extracellularly. ROS are produced in cellular structures such as membranes, lysosomes, and mitochondria. Their formation can be exacerbated by external agents including ultraviolet (UV) light, tobacco, infections and chemicals. The human body protects itself from ROS by utilizing antioxidant enzymes (SOD), such as catalases and glutathione peroxidases, which neutralize radicals and produce less harmful substances. However, as one's body declines with age, levels of these antioxidant enzymes also decrease correspondingly ${ }^{[9]}$.

Alteration of matrix metalloproteinase (MMP) levels can lead to dysregulation of skin homeostasis ${ }^{[10]}$. In the elderly, the levels of zinc-dependent proteases increase, causing degradation of ECM protein components. Physiologically, MMPs ensure protein turnover by stimulating the renewal of elastic and collagen fibers. MMP levels are regulated by tissue inhibitors, the expression of which can be disturbed by UV light, smoke and ROS. Protein-degrading MMPs in contrast, render connective tissue less elastic. Another critical point to be considered is protein glycation, namely the reaction of glucose and other sugars such as fructose. This post translational protein modification is associated with increased levels of glucose, affects protein structure and function through increased cross-linking, and results in the formation of "Amadori" and advanced glycation end products. Glycated proteins increase the formation of free radicals and release toxic products and pro-inflammatory components that cause protein damage, which ultimately is the fundamental cause of aging ${ }^{[1,12]}$. In women, menopause is associated with reduced ovarian endocrine activity leading to an increase in follicular stimulating hormone and decrease in estrogen and progesterone, with downstream metabolic disturbances. Alterations start from sexual organs and involve all tissues targeted by estrogen, in a multisystem framework that affects all connective tissues. Collagen atrophy is the main factor associated with menopause-related skin aging, which contributes to reduced skin elasticity ${ }^{[13]}$. In menopause, skin thickness and collagen content are initially reduced by $1.13 \%$ and $2.1 \%$ per year respectively; type I and III collagen decrease by $30 \%$. The skin is not only a target for sex-hormones but also produces and releases estrogen from the enzymatic conversion of estrogen precursors. Estrogen exerts a number of functions on connective tissue such as counteracting the degradation of collagen by MMPs induced by UV light and ROS through the activation of the TGF- $\beta 1$ pathway. Skin and bone thickness 
Table 1. Extracellular matrix components can be divided into 3 main groups

\begin{tabular}{|c|c|c|c|}
\hline Structural proteins & Adhesion proteins & Glycosaminoglycans & Proteoglycans \\
\hline $\begin{array}{l}\text { Collagen } \\
\text { Elastin }\end{array}$ & $\begin{array}{l}\text { Fibronectin } \\
\text { Fibrillin } \\
\text { Laminin } \\
\text { Tenascine } \\
\text { Vitronectine } \\
\text { Osteonectine }\end{array}$ & $\begin{array}{l}\text { laluronan } \\
\text { Heparan-sulphate } \\
\text { Condroitn-sulphate }\end{array}$ & $\begin{array}{l}\text { Biglican } \\
\text { Agregacan } \\
\text { Versican } \\
\text { Neurocan }\end{array}$ \\
\hline
\end{tabular}

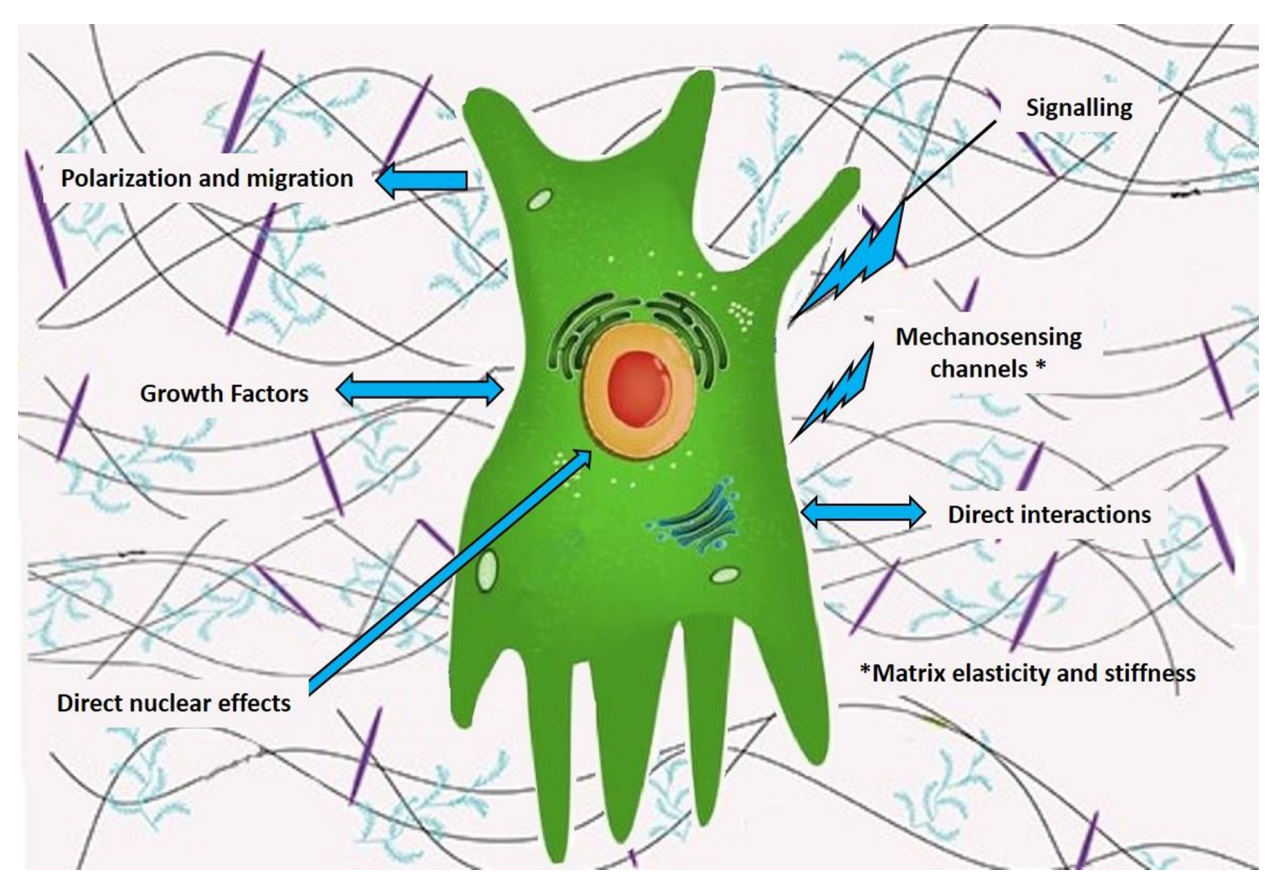

Figure 1. Interactions extracellular matrix cell

are also positively correlated with estrogen levels. Post-menopausal women with less estrogen, show a decreased expression of TGF- $\beta 1$ in skin fibroblasts, which may hamper the ability of fibroblasts to produce collagen, elastin and proteoglycans.

\section{THE MATRISOME: SIGNIFICANCE AND FUNCTION}

In recent years, both antiaging and medical research have focused on ECM biochemistry and pathophysiology as well as matrix dysregulation which can lead to disease progression ${ }^{[14,15]}$. The ECM fills the intercellular space and is present in all connective tissues [Table 1]. Dermis and adipose, muscle, bone, cartilage, and the surrounding parenchyma of organs, are all connective tissue, which highlights the relevance of the ECM in maintaining tissue homeostasis. Within the intercellular space, phenomena like cellular polarization and migration, regulation of growth factors, activation of signaling molecules, and processes translating mechanical stimulation into a chemical signal through the involvement of mechanosensitive channels are all essential for the maintenance of ECM elasticity and physiological tissue stiffness [Figure 1]. Mechano-transduction in particular, is an increasingly well-studied process. When the fibroblast is located in a stiff microenvironment, it will be stimulated continuously to produce new ECM. In addition, basement membranes play a fundamental role in maintaining tissue homeostasis and the transmission of mechano-transduction signals to the underlying dermis.

The molecular structure of the ECM is responsible for the above essential functions. The ECM is also comprised of a complex network of proteins defined as the "matrisome" [Table 1]. The matrisome is now 
a matter of intense study, not only for the identification of all component proteins but also, for defining the molecular mechanisms regulated by the $\mathrm{ECM}^{[16]}$. To date, about 300 matrisome proteins have been identified including: 43 types of collagen subunits in fibrillar and non-fibrillar form (the most common is type 1 representing $80 \%-90 \%$ ); 36 types of proteoglycans dispersed amongst collagen fibers to take up the interstitial space and maintain hydration; growth factors linked to glycosaminoglycans that are retained in the extracellular space to increase functionality; about 200 soluble proteins, which not only promote assembly of the ECM but are also involved in cell-ECM interactions, and act as ligands with receptors (integrins); and enzymes such as lysil-oxidase, transglutaminase and hydroxylase ${ }^{[17]}$. To simplify, the matrisome is composed of 3 main molecular groups: structural proteins, adhesion proteins and proteoglycans. Among structural proteins, collagen is most abundant. Its peculiar amino acid sequence provides essential functions ${ }^{[18]}$. Aging reduces collagen synthesis and alters its structure (collagen fibers become fragmented and stiff). Amino acids are fundamental to the structure and thus, function of collagen - for example, the interaction of specific amino acids with potassium ions favors non-covalent binding and binding to proteoglycans. There is less elastin overall but it is still essential for maintaining the elastic properties of skin. Elastin fibers stretch under traction but with mechanical stress, can be restored to their original conformation. This process is due to the presence of hydrophobic amino acids such as valine and alanine, both of which are exposed to the hydrophilic components of ECM during mechanical stress. When the mechanical force is removed, elastin folds back on itself and its fibers become shortened. It goes without saying that the optimal functionality of elastin would also be dependent on adequate tissue hydration ${ }^{[19]}$.

Therefore, the aging process would undoubtedly influence the ECM and matrisome as a whole. All 300 matrisome proteins are affected and their turnover is related to their individual half-lives. To illustrate, the half-life of collagen is about 3 years and that of elastin is 70, which means that post-translational modifications like glycation have an essential role in skin elasticity and structure. The increase in MMPs is also part of aging and correspondingly, structural proteins such as collagen and elastin lose function and contribute towards a loss of skin tone. The imbalance of peptide content has to be taken into account as well, in terms of cellular cross-talk alterations.

\section{TREATMENT OF ECM ALTERATION IN SKIN AGING}

Given the above discussion, the most advanced antiaging treatments would therefore be those that are able to rebuild the ECM, which is responsible for both physical support and the exchange of nutrients, cellular mediators, and growth factors ${ }^{[2,21]}$. The most effective treatment would target cell metabolism, autophagy, cell renewal and the production of new ECM. Such results can theoretically be obtained by providing the right metabolic and structural support to cells (i.e., hyaluronic acid, specific amino-acids and peptides, antioxidants) as well as mechanical stimulation which would, in turn, stimulate mechano-transduction (that is the conversion of mechanical forces into biochemical signals) through the creation of a scaffold in reduced volumes to enable stimulation of interstitial fluid circulation.

Low and/or high molecular weight, natural (not cross-linked) hyaluronic acid have been reported to be capable of hydrating the ECM, thereby stimulating cellular activity when administered as injectable formulas. Low molecular weight hyaluronic acid has the ability to stimulate cells thereby preventing aging, increasing skin brightness, hydration, tone, reducing wrinkles and restoring volume. On the other hand, high molecular weight forms exert powerful antioxidant activity by neutralizing free radicals and becoming a scaffold for the skin. Hyaluronic acid however, must be continuously produced as they have particularly short half-lives and are degraded by specific enzymes, hyaluronidases. To increase the consistency and longevity of injected hyaluronic acid in the dermis, it is necessary to render it less susceptible to hyaluronidases by chemical modification with cross-linking agents to bridge its filaments. The such chemically-modified hyaluronic acid has a greater filling effect but its biological properties are drastically reduced in favor of the filling capacity (filler) making fillers a different issue (they consist of cross-linked 
hyaluronic acid, made denser and chemically modified by crosslinking agents) which actually fills the tissue with immediate results compared to natural hyaluronic acid, but with lower biological activity ${ }^{[22,23]}$.

It should be highlighted that the use of dermal fillers does not exclude treatment with hyaluronic acid and vice versa. Often, a combination of treatment strategies allows one to easily achieve the desired results ${ }^{[24]}$. Fibrous proteins of the dermis, such as collagen and elastin, are characterized by a specific aminoacid composition. With age, the ability of fibroblasts to synthesize collagen is reduced. Furthermore, alteration of collagen fibers imparts increasing stiffness and decreased elasticity to skin, with a consequent loss of tone and softness. An anti-aging treatment should therefore, always have, among others, the ability to increase the quantity and quality of collagen fibers. This can be achieved through specific cosmetic treatments capable of stimulating the production of collagen (peptides, retinol, vitamin C), food supplements containing specific amino acids or compounds capable of stimulating fibroblasts activity (i.e., collagen, amino acids and specific peptides), and even bio-revitalizing injections. In fact, in the absence of adequate amounts of amino acids, cells react by blocking catabolism. As a result, proteins age and become less efficient. "Old" proteins, whether enzymes, structural or contractile, become increasingly fragile ${ }^{[25]}$.

\section{CONCLUSION}

Various factors associated with an individual's genetic background and physical environment can affect one's skin in different ways with regards to skin aging. Previously, skin aging was thought of as physiological or chrono-aging, and environmental or photo-aging. Other external factor have now been recognized to influence the skin aging process including gravity, environmental pollution, climate, and smoking. In order to provide a personalized treatment plan, a thorough analysis of an individual's aging processes will be required. For example, the shape of the face is of utmost importance - overtime, round faces tend to sag while thin and elongated faces have a tendency to become hollow. In the former, lifting would be required while in the latter, volumizing would be necessary. Treatment choice should be based on demonstrated safety and efficacy while injection technique is very important for delivery and stems from an in-depth knowledge of the anatomy and physiology of the treatment sites ${ }^{[2]}$. To illustrate, by specifically defining injection points or by combining injections with facial lymph node drainage, results will be immediately visible and continue to improve over the ensuing weeks and months ${ }^{[27]}$. Treatment protocols (number of sessions, frequency, maintenance, mix of introduced substances, combination with cosmetics, and nutraceuticals even) have to be individualized in order to suit the patient's needs. Aesthetic dermatology is perhaps the first medical discipline dedicated towards studying the properties of ECM and its treatment but has increasingly, gained the attention of other clinical fields ${ }^{[28]}$ such as orthopedics, dentistry, pulmonology and gastroenterology. We believe that in the near future, the ECM will be recognized as a master element of many organs and body systems, overcome the barriers and concepts of current specialty fields, and improve our mechanistic understanding of the many internal and external dysfunctions and disease states for better treatment.

\section{DECLARATIONS}

\section{Authors' contributions}

The author contributed solely to the article.

\section{Availability of data and materials}

Not applicable.

\section{Financial support and sponsorship}

None.

\section{Conflicts of interest}

The author declared that there are no conflicts of interest. 


\section{Ethical approval and consent to participate}

Not applicable.

\section{Consent for publication}

Not applicable.

\section{Copyright}

(c) The Author(s) 2020.

\section{REFERENCES}

1. Tobin DJ. Introduction to skin aging. J Tissue Viability 2017;26:37-46.

2. Sparavigna A. How does face age? A retrospective observational study and meta-analysis. J Plast Pathol Dermatol 2019;15:1-8.

3. Michaud T, Gassia V, Belhaouari L. Facial dynamics and emotional expressions in facial aging treatments. J Cosmet Dermatol 2015;14:9-21.

4. Glogau RG. Aestethic and anatomic analysis of the aging skin. Semin Cutan Med Surg 1996;15:134-8.

5. Hynes RO. The extracellular matrix: not just pretty fibrils. Science 2009;326:1216-9.

6. Widgerow AD, Fabi SG, Palestine RF, Rivkin A, Ortiz A, et al. Extracellular matrix modulation: optimizing skin care and rejuvenation procedures. J Drugs Dermatol 2016;15:s63-71.

7. Qa'aty N, Vincent M, Wang Y, Wang A, Mitts TF, et al. Synthetic ligands of the elastin receptor induce elastogenesis in human dermal fibroblasts via activation of their IGF-1 receptors. J Dermatol Sci 2015;80:175-85.

8. Widgerow AD, Jiang LI, Calame A. A single-center clinical trial to evaluate the efficacy of a tripeptide/hexapeptide antiaging regimen. J Cosmet Dermatol 2019;18:176-82.

9. Naylor EC, Watson REB, Sherrat MJ. Molecular aspects of skin ageing. Maturitas 2011;69:249-56.

10. Panwar P, Butler GS, Jamroz A, Azizi A, Overall CM, et al. Aging-associated modifications of collagen affect its degradation by matrix metalloproteinases. Matrix Biol 2018;65:30-44.

11. Langton AK, Sherrat MJ, Griffiths CEM, Watson REB. Review article: a new wrinkle on old skin: the role of elastic fibers in skin ageing. Int J Cosmet Sci 2010;32:330-9.

12. Zouboulis CC, Elewa R, Ottaviani M, Fluhr J, Picardo M, et al. Age influences the skin reaction pattern to mechanical stress and its repair level through skin care products. Mech Ageing Dev 2018;170:98-105.

13. Raine-Fenning NJ, Brincat MP, Muscat-Baron Y. Skin aging and menopause: implications for treatment. Am J Clin Dermatol 2003;4:371-8.

14. Theocharis AD, Skandalis SS, Gialeli C, Karamanos NK. Extracellular matrix structure. Adv Drug Deliv Rev 2016;97:4-27.

15. Bonnans C, Chou J, Werb Z. Remodelling the extracellular matrix in development and disease. Nat Rev Mol Cell Biol 2014;15:786-801.

16. Hynes RO, Naba A. Overview of the matrisome - an inventory of extracellular matrix constituents and functions. Cold Spring Harb Perspect Biol 2012;4:a004903.

17. Raghunathan R, Sethi MK, Klein JA, Zaia J. Proteomics, glycomics, and glycoproteomics of matrisome molecules. Mol Cell Proteomics 2019;18:2138-48

18. Shoulders MD, Raines RT. Collagen structure and stability. Annu Rev Biochem 2009;78:929-58.

19. Weihermann AC, Lorencini M, Brohem CA, de Carvalho CM. Elastin structure and its involvement in skin photoageing. Int J Cosmet Sci 2017:39:241-7.

20. Krutmann J, Bouloc A, Sore G, Bernard BA, Passeron T. The skin aging exposome. J Dermatol Sci 2017;85:152-61.

21. Boulter E, Estrach S, Errante A, Pons C, Cailleteau L, et al. CD98hc (SLC3A2) regulation of skin homeostasis wanes with age. J Exp Med 2013;210:173-90.

22. Sparavigna A, Tenconi B, Giori AM, Bellia G, La Penna L. Evaluation of the efficacy of a new hyaluronic acid gel on dynamic and static wrinkles in volunteers with moderate aging/photoaging. Clin Cosmet Investig Dermatol 2019:12:81-90.

23. Sparavigna A, Orlandini A. Efficacy and tolerance of an injectable medical device containing hyaluronic acid and amino acids: a monocentric six-month open label evaluation. J Clin Trials 2017:7:4-12.

24. Sparavigna A, Tenconi B. Efficacy and tolerance of an injectable medical device containing stable hybrid cooperative complexes of highand low-molecular-weight hyaluronic acid: a monocentric 16 weeks open-label evaluation. Clin Cosmet Investig Dermatol 2016;9:297-305.

25. Limbert G, Masen MA, Pond D, Graham HK, Sherratt MJ, et al. Biotribology of the ageing skin - why we should care. Biotribology 2019;17:75-90.

26. Hu S, Li Z, Cores J, Huang K, Su T, et al. Needle-free injection of exosomes derived from human dermal fibroblast spheroids ameliorates skin photoaging. ACS Nano 2019;13:11273-82.

27. Sparavigna A. The interstitial fluid technique and the extracellular matrix. Prime Journal, Case Study, Injectables, Dermatology. Available from https://www.prime-journal.com/the-interstitial-fluid-technique-and-the-extracellular-matrix/ [Last accessed on 19 Mar 2020]

28. Bhattacharjee O, Ayyangar U, Kurbet AS, Ashok D, Raghavan S. Unraveling the ECM-immune cell crosstalk in skin diseases. Front Cell Dev Biol 2019;7:68. 